declared, Kelly Gavigan: None declared, Shilpa Venkatachalam: None declared, Laura Stradford: None declared, Jessica Boles: None declared, Justin Owensby: None declared, Cassie Clinton: None declared, Ilya Lipkovich Shareholder of: El Lilly and Company, Employee of: Eli Lilly and Company, Amy Calvin Shareholder of: Eli Lilly and Company, Employee of: Eli Lilly and Company, Virginia S. Haynes Shareholder of: Eli Lilly and Company, Employee of: Eli Lilly and Company DOI: 10.1136/annrheumdis-2020-eular.2355

\section{THU0565 $\quad$ RESEARCHERS' PERSPECTIVES ON ADHERENCE INTERVENTION RESEARCH AND OUTCOMES IN RHEUMATOLOGY: AN INTERNATIONAL QUALITATIVE STUDY}

S. Salmasi ${ }^{1}$, A. Kelly², S. J. Bartlett ${ }^{3}$, M. De Wit', L. March ${ }^{5}$, A. Tong ${ }^{5}$, P. Tugwell ${ }^{6}$, K. Tymms ${ }^{2}$, S. Verstappen ${ }^{7}, \mathrm{M}$. De Vera ${ }^{1}$ on behalf of OMERACT-Adherence Working Group. ${ }^{1}$ University of British Columbia, Vancouver, Canada; ${ }^{2}$ The Australian National University, Canberra, Australia; ${ }^{3}$ Mc Gill University, Montréal, Canada; ${ }^{4}$ OMERACT Patient Research Partner, Amsterdam, Netherlands; ${ }^{5}$ The University of Sydney, Camperdown, Australia; ${ }^{6}$ University of Ottawa, Ottawa, Canada; ${ }^{7}$ The University of Manchester, Manchester, United Kingdom

Background: Medication non-adherence is a significant problem among patients with rheumatic diseases. Research on adherence interventions in rheumatology is limited and disappointing, with studies using heterogeneous outcomes. Understanding these limitations is needed to inform the design of better interventions and research studies.

Objectives: To describe researchers' perspectives and experiences on adherence intervention research and outcomes in rheumatology.

Methods: Semi-structured interviews using video conference were conducted with researchers who had been an investigator on an adherence study of any design in the past 10 years. Interviews were recorded and transcribed verbatim. Participants were asked about their experiences with conducting adherence research and perspectives on introduction of a core domain set of outcomes for adherence intervention trials in rheumatology. Data collection and thematic analysis were conducted iteratively, until saturation.

Results: We interviewed 13 researchers from seven countries (Australia, Belgium, Canada, Netherland, Thailand, UK, and USA). A majority worked in academia $(75 \%)$, specialized in epidemiology and/or health services research $(62 \%)$ and had led between 2-5 adherence studies in the past five years (62\%)

Three themes were identified: 1) challenges in designing, conducting and evaluating adherence studies; 2) current outcomes in adherence intervention studies and their relevance; and 3) implementing a core domain set of outcomes for adherence intervention studies.

Major challenges in conducting adherence research included inconsistent adherence terminology and measurement. Participants noted a lack of guidance on outcome selection and measurement when evaluating the effectiveness of an adherence intervention and indicated their preference for research to report adherence, intervention-specific, and health-related outcomes. Finally, implementing a core domain set of outcomes was thought to be challenging but valuable in strengthening the evidence (by facilitating meta-analysis), and improving clinical outcomes (by informing clinicians about the effectiveness of interventions).

Conclusion: Adherence research in rheumatology has been hindered by lack of standardization and guidance on terminology, measurement and outcome selection. Our findings form the basis for recommendations for improving the design, conduct and evaluation of adherence intervention studies in rheumatology, particularly for developing a core domain set of outcomes to improve consistency and facilitate comparisons.

Table 1. Themes and representative quotations.

Theme 1: Challenges in designing, conducting and evaluating studies of adherence interventions

“...the people you often most want in your sample are the people who are non-adherent and often the people who are non-adherent are the people who are hardest to recruit"

"Long term the issue has been about measurements because people confuse and conflate various aspects of medication adherence.

Theme 2: Current outcomes in adherence intervention studies and their relevance "you have a whole range of outcomes...psychological outcomes...there's measures of health care utilization and things like attendance at hospital, nurse appointments and duration, things like times off work, and also all the relevant clinical outcomes."

Theme 3: Implementing a core domain set of outcomes for adherence intervention studies

"...will make trials more comparable and increase the likelihood that you'd be able to combine efforts internationally"
Disclosure of Interests: Shahrzad Salmasi: None declared, Ayano Kelly: None declared, Susan J. Bartlett Consultant of: Pfizer, UCB, Lilly, Novartis, Merck, Janssen, Abbvie, Speakers bureau: Pfizer, UCB, Lilly, Novartis, Merck, Janssen, Abbvie, Maarten de Wit Grant/research support from: Dr. de Wit reports personal fees from Ely Lilly, 2019, personal fees from Celgene, 2019, personal fees from Pfizer, 2019 , personal fees from Janssen-Cilag, 2017, outside the submitted work., Consultant of: Dr. de Wit reports personal fees from Ely Lilly, 2019, personal fees from Celgene, 2019, personal fees from Pfizer, 2019, personal fees from Janssen-Cilag, 2017, outside the submitted work., Speakers bureau: Dr. de Wit reports personal fees from Ely Lilly, 2019, personal fees from Celgene, 2019, personal fees from Pfizer, 2019, personal fees from Janssen-Cilag, 2017, outside the submitted work., Lyn March None declared, Allison Tong: None declared, Peter Tugwell: None declared, Kathleen Tymms: None declared, Suzanne Verstappen Grant/research support from: BMS, Consultant of: Celltrion, Speakers bureau: Pfizer, Mary De Vera: None declared DOI: 10.1136/annrheumdis-2020-eular.4787

THURSDAY, 04 JUNE 2020

\section{Rehabilitation}

\begin{tabular}{l|l}
\hline THU0566 & THE EFFECT OF KINESIO-TAPING ON CHRONIC \\
NONSPECIFIC LOW BACK PAIN: PRELIMINARY \\
RESULTS OF A DOUBLE BLINDED RANDOMIZED \\
CLINICAL TRIAL.
\end{tabular}

I. Aachari ${ }^{1}$, K. Samia ${ }^{2}$, T. Latifa ${ }^{1}$, T. Fatima Zahrae ${ }^{1}$, S. Afilal ${ }^{1}$, F. Safaa ${ }^{1}$, T. El Joumani $^{1}$, S. Bahloul ${ }^{1}$, H. Rkain ${ }^{1,3}$, I. Hmamouchi ${ }^{4}$, R. Abouqal ${ }^{4}$, F. Allali ${ }^{1} .{ }^{1} E I$ Ayachi Hospital, Rheumatology, Salé, Morocco; ${ }^{2}$ El Ayachi Hospital, Physical Medicine and Rehabilitation, Salé, Morocco; ${ }^{3}$ Faculty of Medicine and Pharmacy, Mohamed V University, Laboratory of Physiology, Rabat, Morocco; ${ }^{4}$ Faculty of Medicine and Pharmacy, Mohamed V University, Laboratory of Epidemiology and Clinical Research, Rabat, Morocco

Background: The technique of Kinesio-Taping is a method of adhesive bandage exerting traction on the skin which would favorably influence the muscular and articular systems by reducing the pressure exerted on the subcutaneous mechanoreceptors thus reducing pain and muscle tension.

Objectives: The aim of this study is to assess the effectiveness of Kinesio-Taping in the short and medium term on pain and function in patients with chronic nonspecific low back pain compared to a placebo.

Methods: We conducted a double-blind, two-arm randomized clinical trial. The study should include a total of 70 patients randomized into 2 groups: Kinesio-Taping $(n=35)$ and control group $(n=35)$. To this date we have included 46 patients All patients receives four I-shaped adhesive strips arranged in a star-like shape and applied to the most painful region of the lower back with a tension between $25 \%$ to $30 \%$ in the taping group. The placebo group received a taping procedure with no tension. Taping is applied three times (at baseline, fourth and eighth day). Patients are assessed at baseline, on day 14 and at 4 weeks by the Arabic version of the Oswestry Physical and Functional Disability Index (ODI) which is the primary outcome. The secondary outcomes are the assessment of pain and functional disability according to the visual analog scale (VAS) evaluated on a scale of 0 to 10, as well as Rolland-Morris score.

Results: Both groups were comparable at baseline concerning the demographical and clinical characteristics $(P>0.05)$ (table 1). The result of repeated measures ANOVA showed a significant change in ODI score and in VAS for pain and functional disability as well as Rolland-Morris score in both groups. Using the ANCOVA, controlling for pre-test scores, a significant difference was found between the two groups (table 2).

Table 1. Clinical characteristics of study population.

\begin{tabular}{|l|c|c|c|}
\hline & Taping groupe $(\mathrm{n}=22)$ & Placebo groupe $(\mathrm{n}=24)$ & $p$ value \\
\hline Age & $\mathbf{4 2} \pm \mathbf{8 , 3 2}$ & $\mathbf{4 0} \pm 9, \mathbf{5 6}$ & $\mathbf{0 , 6 1 8}$ \\
\hline Female gender & $\mathbf{2 1}$ & $\mathbf{1 8}$ & $\mathbf{0 , 0 9 8}$ \\
\hline Prolonged standing position & $\mathbf{1 4}$ & $\mathbf{1 6}$ & $\mathbf{0 , 8 2 9}$ \\
\hline Heavy load carrying & $\mathbf{1 5}$ & $\mathbf{1 6}$ & $\mathbf{0 , 9 1 3}$ \\
\hline Walking aid & $\mathbf{0}$ & $\mathbf{2}$ & $\mathbf{0 , 4 9 0}$ \\
\hline Comorbidities & $\mathbf{8}$ & $\mathbf{6}$ & $\mathbf{0 , 4 0 3}$ \\
\hline Body mass index & $\mathbf{2 7} \pm \mathbf{3 , 6 8}$ & $\mathbf{2 8} \pm \mathbf{7 , 0 2}$ & $\mathbf{0 , 5 0 1}$ \\
\hline ODI of baseline & $\mathbf{3 6} \pm \mathbf{1 8 , 8 2}$ & $\mathbf{3 1} \pm \mathbf{1 2 , 6 5}$ & $\mathbf{0 , 3 2 9}$ \\
\hline
\end{tabular}


Conclusion: Our clinical trial offers preliminary evidence on the superiority of Kinesio-Taping in the treatment chronic back pain compared to placebo concerning the reduction of pain and disability. Thus, it can be used as a complementary method in chronic non-specific low back pain.

Table 2. Primary and secondary outcomes in the Kinesio-Taping and placebo group.

\begin{tabular}{|c|c|c|c|c|c|c|c|c|c|}
\hline & \multicolumn{4}{|c|}{ Taping groupe ( $\mathrm{n}=22$ ) } & \multicolumn{4}{|c|}{ Placebo groupe $(\mathrm{n}=24)$} & \multirow{2}{*}{ ANCOVA } \\
\hline & Baseline & Day 12 & 4 weeks & $p$ value & Baseline & Day 12 & 4 weeks & $p$ value & \\
\hline ODI & $36 \pm 18,82$ & $24 \pm 19,83$ & $24 \pm 19,32$ & $<0,001$ & $31 \pm 12,65$ & $28 \pm 13,32$ & $29 \pm 13,24$ & 0,03 & $<0,001$ \\
\hline VAS of pain & $7 \pm 1,3$ & $3,9 \pm 2,2$ & $4,6 \pm 2,2$ & $<0,001$ & $6,5 \pm 1,2$ & $5,5 \pm 2,2$ & $5,7 \pm 2,2$ & 0,008 & $<0,001$ \\
\hline $\begin{array}{l}\text { VAS of } \\
\text { functional } \\
\text { disability }\end{array}$ & $6,7 \pm 1,5$ & $4 \pm 2,8$ & $4,4 \pm 2,5$ & $<0,001$ & $5,9 \pm 2,3$ & $5,2 \pm 2,2$ & $5,3 \pm 2,2$ & 0,003 & $<0,001$ \\
\hline $\begin{array}{l}\text { Rolland } \\
\text { Morris }\end{array}$ & $7,4 \pm 4,6$ & $5,2 \pm 4,7$ & $5,3 \pm 4,6$ & $<0,001$ & $8,4 \pm 4,8$ & $7,6 \pm 4,2$ & $7,3 \pm 4,1$ & 0,05 & 0,06 \\
\hline
\end{tabular}

Disclosure of Interests: None declared

DOI: 10.1136/annrheumdis-2020-eular.2979

\section{THU0567 HIP ABDUCTORS STRENGTH AND TRUNK, PELVIS, HIP AND KNEE FRONTAL PLANE KINEMATICS ANALYSIS DURING SINGLE-LEG SQUAT IN INDIVIDUALS WITH AND WITHOUT PATELLOFEMORAL OSTEOARTHRITIS}

C. Carvalho $^{1}$, G. Keppe Pisani ${ }^{1}$, A. Felipe Martinez ${ }^{1}$, L. Mancini ${ }^{1}$, F. Viadanna Serrão ${ }^{1}$, P. Regina Mendes Da Silva Serrão ${ }^{1} .{ }^{1}$ Federal University of São Carlos, Physical Therapy, São Carlos, Brazil

Background: Previous studies have observed that individuals with patellofemoral pain (PFP) have decreased hip abduction torque, as well as increased hip adduction and knee abduction during activities with uniltateral weight bearing ${ }^{1,2}$ Considering that, a significant number of patients with patellofemoral osteoarthritis (PFOA) have a previous history of $\mathrm{PFP}^{3}$, it is speculated that the mechanical causes of PFP and PFOA may be similar. However, although alterations in hip muscle strength and lower limb kinematics during various functional activities have been reported in patients with PFP, they have not been explored in subjects with PFOA.

Objectives: The objectives of this study were to compare the hip eccentric abductors torque and the trunk, pelvis, hip, and knee frontal plane kinematics in subjects with and without PFOA isolated during the single-leg squat.

Methods: This is a cross-sectional study. The volunteers were divided into two groups: control group (CG - healthy individuals) and PFOA group (PFOAG - individuals with PFOA grade II or III). Eccentric peak torque of the hip abductors was evaluated using an isokinetic dynamometer Biodex Multi-Joint System 3, at angular speed of $30 \%$ s. Trunk, pelvis, hip and knee kinematics were recorded during the single-leg squat using a 6-camera, 3-dimensional motion-analysis system (Vicon Motion Systems, Nexus System 2.1.1 and 3D Motion Monitor). The t-test Student was used to compare the variables between the groups. The significance level was set at $5 \%$ for all analyses ( $\mathrm{p} \leq 0.05$ ).

Results: The CG was composed by 12 participants (41.7\% women). PFOA had 9 participants $(44.4 \%$ women). Age $(p=0.1)$, height $(p=0.9)$ and body mass $(p=0.2$ ) showed homogeneity between groups. Regardind body mass index, the OAPFG showed higher values $(p=0.02)$. PFOAG showed greater increased

Table 1 - Values of joint angles during single-leg squatting and hip abductors torque (normalized by body mass) in PFOA and healthy individuals

\begin{tabular}{lccc}
\hline & PFOAG & CG & $p$ value \\
\hline Contralateral trunk lean $\left(^{\circ}\right)$ & $4.7 \pm 1.3$ & $4.8 \pm 1.1$ & 0.94 \\
Contralateral pelvic elevation $\left(^{\circ}\right)$ & $1.4 \pm 1.2$ & $2.6 \pm 1.0$ & 0.45 \\
Hip adduction $\left({ }^{\circ}\right)$ & $14.9 \pm 2.2$ & $8.9 \pm 1.6$ & $0.05^{*}$ \\
Knee abduction $\left({ }^{\circ}\right.$ ) & $22.8 \pm 3.8$ & $19.4 \pm 3.3$ & 0.51 \\
Hip eccentric abdutor torque (Nm/kg.100) & $166.5 \pm 24.9$ & $204 \pm 27.7$ & $0.006^{*}$
\end{tabular}

PFOAG: patellofemoral osteoarthritis group; CG: control group *Statistical difference between groups hip adduction than CG $(p=0.05)$ (Table 1). However, there were no differences among groups for trunk lean, contralateral pelvic elevation and knee abduction $(p>0.05)$. PFOAG showed lower hip abductor torque compared with the CG $(\mathrm{p}=0.006)$.

Conclusion: PFOA individuals showed greater hip adduction and lower hip abductors torque than the CG. Thus, it is suggested that muscle weakness may excessively influence hip adduction. Hip adduction is the main component of the knee valgus in the frontal plane. So, excessive dynamic valgus results in an increase Q-angle and, consequently, an increase in the latera forces acting on the patella, causing greater stress on the lateral patellofemoral joint, which may contribute to disease progression. Therefore, we suggest that the hip abductor strengthening should be considered when treating individuals with PFOA.

References:

[1] Nakagawa TH, Moriya ÉTU, Maciel CD, Serrão FV. Trunk, Pelvis, Hip, and Knee Kinematics, Hip Strength, and Gluteal Muscle Activation During a Single-Leg Squat in Males and Females With and Without Patellofemoral Pain Syndrome. J Orthop Sport Phys Ther. 2012;42(6):491-501.

[2] Nakagawa TH, Moriya ÉTU, Maciel CD, Serrão FV. Frontal Plane Biomechanics in Males and Females with and without Patellofemoral Pain. Med Sci Sport Exerc. 2012;44(9):1747-1755.

[3] Utting MR, Davies G, Newman JH. Is anterior knee pain a predisposing factor to patellofemoral osteoarthritis? Knee. 2005;12(5):362-365.

Acknowledgments: São Paulo Research Foundation (FAPESP) (Grant/Award Numbers: 2017/20057-8; 2017/25959-0; 2018/10329-3).

Disclosure of Interests: None declared

DOI: 10.1136/annrheumdis-2020-eular.5924

\section{THU0568 EFFECTIVENESS OF FOOT ORTHOSIS TO PROMOTE PHYSICAL ACTIVITY FOR PATIENTS WITH CONCURRENT RHEUMATOID ARTHRITIS AND SARCOPENIA}

N. Hishikawa ${ }^{1}$, S. Toyama ${ }^{1,2,3}$, S. Ohashi ${ }^{1}$, K. Sawada ${ }^{1}$, K. Ikoma ${ }^{3}$, D. Tokunaga ${ }^{1}$, Y. Mikami ${ }^{1} .{ }^{1}$ Graduate School of Medical Science, Kyoto Prefectural University of Medicine, Department of Rehabilitation Medicine, Kyoto, Japan; ${ }^{2}$ Graduate School of Medical Science, Kyoto Prefectural University of Medicine, Department of Multidisciplinary Promote for Physical Activity, Kyoto, Japan; ${ }^{3}$ Graduate School of Medical Science, Kyoto Prefectural University of Medicine, Department of Orthopaedics, Kyoto, Japan

Background: Sarcopenia is a progressive systemic skeletal muscle disorder associated with an increased likelihood of adverse outcomes including physical disability, falls, and mortality. The muscle mass of patients with rheumatoid arthritis (RA) is lower than that of age-matched healthy individuals, and a high prevalence rate of sarcopenia has been reported ${ }^{1}$. In particular, foot deformities may increase the prevalence rate of sarcopenia because of inactivity due to foot pain on walking. Treatment with a foot orthosis (FO) can reportedly reduce pain ${ }^{2)}$; however, whether a FO can resolve inactivity and sarcopenia is unclear.

Objectives: To elucidate the effectiveness of a FO on physical activity and sarcopenia in patients with RA.

Methods: Thirty patients with RA with foot deformities were enrolled from April 2017 to December 2019. Sarcopenia was diagnosed using the algorithm of the European Working Group on Sarcopenia in Older People, and the cut-off values of the Asian Working Group for Sarcopenia were applied. We also collected the clinical variables of patients with concurrent RA and sarcopenia who continued to use a FO for 6 months. The primary outcome was physical activity determined by the International Physical Activity Questionnaire. The secondary outcomes were foot pain measured with a visual analog scale; activities of daily living (ADL) measured with the Health Assessment Questionnaire; and body mass index, body fat percentage, and the skeletal muscle mass index measured with a body composition device. The clinical variables were compared between baseline and 6 months after continuous treatment with a FO.

Results: The prevalence rate of sarcopenia was $76.6 \%(23 / 30)$, and nine patients with RA continued to use the FO for 6 months. Table 1 shows outcomes at baseline and after 6 months of treatment with a FO. The only clinical variable that showed a significant difference was foot pain Physical activities, ADL, and body compositions were maintained after 6 months. 